Katharina Reiss

\title{
Adäquatheit und Äquivalenz
}

\begin{abstract}
The science of translation theory, like any other science, is in need of specific terminology which is commonly accepted as regards definition and content description. Translation theory very often makes use of two concepts, in particular: "Adäquatheit" and "Äquivalenz", though without defining these terms in an unambiguous way. This paper attempts to delimit one concept in relation to the other and to provide definitions accordingly.
\end{abstract}

Wie jede Wissenschaft so braucht auch die Übersetzungswissenschaft ihre Fachtermini; und wenn eine Didaktik des Übersetzens mit Aussicht auf Erfolg entwickelt werden soll, so ist es nicht nur wünschenswert sondern unabdingbar, daß über die wichtigsten Fachtermini Konsens herrscht, eine allgemein anerkannte Definition oder zumindest eine plausible Beschreibung der Schlüsselbegriffe zur Verfügung stehen.

In der Literatur zu Fragen des Übersetzens, der Übersetzungskritik und der Übersetzungsdidaktik tauchen nun immer wieder die Begriffe Adäquatheit und Äquivalenz auf, ohne daß sie in sich eindeutig definiert oder gegeneinander ausreichend abgegrenzt wären, obwohl es sich bei ihnen um Schlüsselbegriffe der Translatologie handelt. Im folgenden soll versucht werden, bei aller gebotenen Kürze die beiden Begriffe - die ja gelegentlich sogar als Synonyme verwendet werden (Stackelberg 1978, 8) - voneinander abzugrenzen und sie zu definieren.

\section{Adäquatheit}

Adäquatheit ist so viel wie Angemessenheit. Angemessenheit ist keine Größe in sich, sondern muß im Zusammenhang mit einem Tun gesehen werden. Man kann etwas angemessen tun im Blick auf den Zweck des Handelns. Da der die jeweiligen Entscheidungen beherrschende Faktor beim Übersetzen der Zweck einer Übersetzung ist, müssen die übersetzerischen Entscheidungen diesem Zweck angemessen sein. Adäquatheit ist also eine Relation Mittel: Zweck und ist damit prozeßorientiert (handlungsorientiert).

Äquivalenz = Gleichwertigkeit dagegen ist eine Relation zwischen zwei Produkten - dem Ausgangs- und dem Zieltext -. Die Notwendigkeit, beide Begriffe scharf voneinander zu trennen, ergibt sich aus dem Umstand, daß Übersetzungen unterschiedlichen Zwecken dienen können - 
auch anderen Zwecken als der Ausgangstext - und daß davon die Äquivalenz der Produkte betroffen wird. Wenn schon nur die verschiedenen Übersetzungstypen berücksichtigt werden, die theoretisch und praktisch existieren, wird dies einsichtig.

Für eine Interlinearversion (Wort-für-Wort-Übersetzung), die einen Ausgangstext Wort für Wort wiedergibt, wählt der Übersetzer, dem Zweck der Übersetzung entsprechend, die angemessenen Worte in der Zielsprache. Wort-Äquivalenz ist mit der adäquaten Wortwahl verbunden, Textäquivalenz jedoch nicht, denn ein Text besteht aus mehr als nur aus isolierten Wörtern. (Funktion dieses Übersetzungstyps: Erforschung noch unbekannter Sprachen. Historisch gesehen, bei den frühen Bibelübersetzern, weil für sie der Text bis in die Wortstellung hinein unantastbar war.)

Für eine wörtliche Übersetzung (auch: grammar translation) wählt der Übersetzer die angemessenen Worte und den angemessenen Satzbau in der Zielsprache. Die adäquate Wahl erzielt lexikalische und grammatische Äquivalenz auf der Satzebene, aber nicht unbedingt Textäquivalenz, denn der Text besteht nicht aus isolierten Einzelsätzen. (Funktion dieses Übersetzungstyps: Kontrollfunktion im Fremdsprachenunterricht).

Für eine philologische Übersetzung wählt der Übersetzer die angemessenen Worte, den angemessenen Satzbau, die angemessene Stilebene in der Zielsprache in engster Anlehnung an die Ausgangssprache. Die Adäquatheit der Sprachzeichenwahl ist dabei also ausgangstextorientiert, denn seit Schleiermacher versteht man unter Adäquatheit bei diesem Übersetzungstyp, daß "der Leser zum Autor geführt" wird, oder wie Ortega es ausdrückt, daß man im Zieltext die Sprach- und Denkstrukturen des Ausgangsautors erkennen kann. Ausgangstextadäquatheit erzielt noch keine Textäquivalenz, weil der Zieltext jetzt vom zielsprachlichen Leser nicht auf eine ebenso natürliche Weise rezipiert werden kann wie der Ausgangstext vom Ausgangsleser. (Funktion: Verständnishilfe für jemanden, der die AS nicht ausreichend beherrscht, um alle ihre Feinheiten zu erkennen).

Der kommunikative Übersetzungstyp erfaßt Übersetzungen ohne gewollte Verfremdungen in Wortwahl und Satzbau; Übersetzungen, die in der zielsprachlichen Gemeinschaft unmittelbar der (alltäglichen, literarischen, künstlerisch-ästhetischen und persuasiven) Kommunikation dienen und dabei mit dem Original in möglichst vielen seiner Dimensionen - der syntaktischen, der semantischen und der pragmatischen - zwar nicht identisch, wohl aber ihm äquivalent sind. Nur in diesem Fall dient die 
Angemessenheit, die Adäquatheit der Sprachzeichenauswahl für den Aufbau des Zieltextes der Herstellung von Äquivalenz auf der Textebene. Und deshalb betrifft hier die Adäquatheit nicht isoliert die Wortwahl, die grammatische und die stilistische Wahl, sondern die adäquate Wahl berücksichtigt immer auch den sprachlichen Makrokontext, den inneren und äußeren Situationskontext und die soziokulturelle (historische) Einbettung des jeweiligen Textes, sowie die Funktion des Gesamttextes im Kommunikationsgeschehen.

Ähnlich wie bei den hier aufgeführten Übersetzungstypen - die sich jeweils durch Dominantsetzung von Teildimensionen eines Ausgangstextes voneinander unterscheiden - verlaufen die Überlegungen, wenn zwar der Text als Ganzes gesehen wird, aber beim Übersetzungsprozeß der Ausgangstext in irgendeiner Weise bewußt bearbeitet wird, weil der Zieltext anderen Zwecken dienen soll als der Ausgangstext. Adäquatheit in der Zeichenwahl richtet sich jetzt nach dem Zweck der Bearbeitung. Ein Übersetzungsprozess liegt auch der Bearbeitung zugrunde, auf Äquivalenz zwischen Ausgangs- und Zieltext wird von vornherein verzichtet.

\section{2. Äquivalenz}

Während Adäquatheit also die zielorientierte Sprachzeichenauswahl im Blick auf einen mit der Übersetzung verfolgten Zweck ist (der nicht derselbe Zweck sein muß, dem der Ausgangstext dienen sollte), ist Äquivalenz die Relation der Gleichwertigkeit von Sprachzeichen in jeweils zwei Sprachsystemen (= der langue-orientierte Äquivalenzbegriff der Kontrastiven Linguistik), und Textäquivalenz ist die Relation der Gleichwertigkeit von Sprachzeichen eines Textes in je zwei verschiedenen Sprachgemeinschaften mit ihrem je eigenen sozio-kulturellen Kontext (der parole-orientierte Äquivalenzbegriff der Übersetzungswissenschaft).

(1) And this is the belief that moves mountains. (J. Webster)

(1a) Und dies ist der Glaube, der Berge bewegt. (Ü.: M. Boveri)

ist eine auf Wort- und Satzebene äquivalente Übersetzung, die Sprachzeichenwahl ist im Satzrahmen adäquat. Textäquivalent wäre jedoch erst die Übersetzung

(1b) Und das ist der Glaube, der Berge versetzt. (Ü.: Boesch-Frutiger),

weil hier berücksichtigt wird, daß in der ZS-Gemeinschaft das Bibelzitat in dieser Form tradiert ist (sozio-kultureller Kontext).

\subsection{Herkunft des Begriffs}


Es herrscht keine Einigkeit darüber, aus welcher anderen Fachsprache der Begriff der Äquivalenz in die Fachsprache der Translationswissenschaft übernommen wurde. W. Wilss $(1977,159)$ vermutet, daß er aus der mathematischen Fachsprache übernommen wurde, wo man von Äquivalenz spricht, "wenn zwischen den Elementen (zweier) Mengen eine umkehrbar eindeutige Zuordnung möglich ist". Für die Maschinenübersetzung, bei der es tatsächlich darum geht, 1:1-Entsprechungen für alle Elemente eines Ausgangstextes festzulegen, mag das hingehen. Für die Humantranslation umkehrbar eindeutige 1:1-Entsprechungen als Äquivalenzmaßstab anzusetzen, ist schlichtweg unmöglich; dagegen spricht u.a. die Variabilitätsorientiertheit aller Sprachen, die unterschiedlichen situationsgebundenen Sprachverwendungsgewohnheiten (Normen) zweier Sprachgemeinschaften und vieles andere mehr. Wenn man sich schon bei anderen Wissenszweigen nach ihrer Definition von Äquivalenz umsieht, scheint mir eine Anleihe bei der Elektrotechnik eher angebracht, wo es heißt: "Zwei Wechselstromschaltungen, die trotz verschiedenen Aufbaus des Netzwerkes für alle Frequenzen gleiches elektrisches Verhalten nach außen zeigen, heißen äquivalent" (Großer Brockhaus). Ohne diese Analogie allzusehr strapazieren zu wollen, läßt sich diese Definition doch eher auf die Translation und Translate übertragen. Der "verschiedene Aufbau des Netzwerks" läßt sich vergleichen mit den texttyp-, textsorten- und textindividuell bedingten Sprachverwendungsweisen in Original und Übersetzung, deren Unterschiede durch die unterschiedlichen Strukturen zweier Sprachen und durch je verschiedene situationelle und kulturelle Bedingungen hervorgerufen werden. Dazu einige Beispiele:

(2) Having entered his house, he unlocked all doors. (Wilss 1977, 204)

(2a) Nachdem er sein Haus betreten hatte ...

(2b) Nach Betreten seines Hauses ...

(2c) Er hatte sein Haus betreten und ... etc.

Hier ergeben sich drei und mehr Möglichkeiten für die Übersetzung, die aufgrund der unterschiedlichen Sprachstrukturen notwendigerweise vom Original abweichen.

(3) El Mundial '82 tendrá lugar en nuestro país. (ABC)

(3a) Die Fußballweltmeisterschaft wird 1982 in Spanien ausgetragen,

muß der deutsche Übersetzer, aufgrund des unterschiedlichen Situationskontextes, schreiben.

(4) Wird der Kompaß von oben abgelesen, so weicht das Ergebnis um $180^{\circ} \ldots \mathrm{ab}$. (Aus einer Gebrauchsanweisung) 
(4a) (Si vous lisez la boussole d'en haut, le résultat déviera de $180^{\circ}$...)

wäre sprachstrukturell möglich, doch die im Französischen üblichen Textsortenkonventionen (Gebrauchsanleitung) fällen die Entscheidung zugunsten von

(4b) Si on lit la boussole ...

(5) Jimmy McBride was dressed as Santa Claus. (Webster)

(5a) Jimmy McBride war als Weihnachtsmann verkleidet. (Ü.: M. Boveri)

Und nicht als St. Nikolaus (Ü.: Boesch-Frutiger), denn es handelt sich um den 24. und nicht um den 6. Dezember (sozio-kultureller Kontext).

Das gleiche elektrische Verhalten, das die beiden Wechselstromschaltungen trotz unterschiedlichen Netzaufbaus zeigen, läßt einen Vergleich mit dem erwünschten gleichen kommunikativ-funktionalen Wirkungsgrad von Original und Übersetzung(en) zu. Das Bild vom Netzwerk erweist sich auch als hilfreich, wenn man nun den Äquivalenzbegriff näher untersucht.

\subsection{Differenzierung des Äquivalenzbegriffs}

Äquivalenz läßt sich beschreiben als Relation zwischen einzelnen sprachlichen Zeichen eines Textpaares und auch als Relation zwischen ganzen Texten. Wenn Äquivalenzbeziehungen zwischen einzelnen Elementen eines Textpaares bestehen, so heißt das noch nicht, daß auch Textäquivalenz insgesamt gegeben ist, das haben einige der bisherigen Beispiele bereits gezeigt. Umgekehrt heißt das aber auch: wenn Textäquivalenz insgesamt gegeben ist, so heißt das noch nicht, daß Äquivalenz zwischen allen Textsegmenten bzw. -elementen eines Textpaares besteht.

Unter der Voraussetzung, daß bei der Translation die Relation der Äquivalenz auf Textäquivalenz zu beziehen ist und diese nur dann realisiert werden soll (bzw. realisiert ist), wenn die beiden in Übersetzungsrelation zueinander gestellten (bzw. stehenden) Texte im Kommunikationsgeschehen der beiden involvierten Sprachgemeinschaften gleichwertige Funktionen erfüllen sollen, muß nun untersucht werden, worin die Textäquivalenz besteht (bzw. festgestellt und beurteilt werden kann).

(6) Is life worth living? - It depends upon the liver!

(6a) La vie, vaut-elle la peine? - C'est une question de foi(e)!

Ein englisches Wortspiel, bei dessen Übersetzung ins Französische keine Äquivalenz auf der Wortebene besteht, das aber volle Textäquiva- 
lenz beanspruchen kann, denn es kann in der französischen Sprachgemeinschaft dieselbe kommunikative Funktion - ein mehr oder weniger geistreiches Wortspiel zu sein - erfüllen. Auf einer höheren Abstraktionsstufe haben beide Texte dieselben Elemente: Frage nach dem Wert des Lebens; Abhängigkeit der Antwort auf diese Frage von einem andern Element; Wortspiel, das die jeweilige Sprachstruktur ausnützt: living/liver auf zwei Zeilen verteilt; foi(e) Homophonie beim Pointenwort. Versucht man, das Wortspiel ins Deutsche zu übersetzen, so fallen mir allein drei Versionen ein:

(6b) Ist das Leben lebenswert? - Das hängt von den Leberwerten ab.

Das kommt auf die Leberwerte an.

Das ist eine Frage der Leberwerte.

Die gemeinsamen Elemente sind wieder die gleichen wie beim englisch/französischen Textpaar; das Wortspiel ist sprachstrukturell bedingt abgewandelt (bezieht jedoch im Unterschied zum Original einen medizinischen Fachausdruck ein), und gründet sich wie im Englischen auf Klangähnlichkeit beim Pointenwort; es umfaßt aber nicht nur ein Textelement (living/liver; foi(e)), sondern bezieht das Element "Wert" mit ein (lebenswert/Leberwerte).

Drei Folgerungen lassen sich aus dem Beispiel ableiten: 1. Die Möglichkeit, Äquivalenz zwischen Ausgangs- und Zieltext herzustellen, wird von den unterschiedlichen Sprachstrukturen beeinflußt und gesteuert und kann in verschiedenen Sprachenpaaren zu unterschiedlichen Lösungen führen; 2. die Einschätzung von Äquivalenz ist auch eine Frage der Bewertung (welche der drei Lösungen bei 6b ist die beste? und gibt es eine beste Lösung?); 3. Äquivalenz ist keine 1:1-Entsprechung, zu der es keine Alternative gibt. Mehrere Übersetzungen können äquivalent sein.

\subsection{Variabilität des Äquivalenzbegriffs}

Darüber hinaus ist festzustellen, daß Äquivalenz in der Translationstheorie überhaupt kein statischer Begriff ist. Es handelt sich um einen flexiblen Begriff, was mit der Natur der Translation und der geschichtlich variablen Auffassungen vom Übersetzen zusammenhängt. Ein Original wird einmal geschrieben/publiziert. Übersetzungen können immer wieder (neu) und von verschiedenen Übersetzern angefertigt werden. Dabei kann sich die Auffassung vom Ausgangstext inzwischen geändert haben; der AS-Text kann von verschiedenen Übersetzern ganz oder in Teilen unterschiedlich interpretiert werden (wobei die Interpretations"bandbreite" durchaus wieder textindividuell und/oder textsortenabhäng- 
ig ist); der Zeitgeschmack kann von einer Übersetzung bestimmte Eigenschaften fordern (vgl. Toury 1980, zu "Übersetzungsnormen"). Die Luther-Bibel etwa mag im 16. Jahrhundert als äquivalenter Zieltext gegolten haben - heute würden wir ihr keine Äquivalenz für unsere Zeit mehr zumessen; zu sehr hat sich der deutsche Sprachgebrauch inzwischen geändert. Z.B. bedeutet das Lexem "Einsamkeit" bei Luther das, was wir heute eher mit "Zweisamkeit" bezeichnen würden; das Lexem "Weiber" hat inzwischen pejorative Konnotationen etc.

Die Übersetzung antiker Dramen im französischen 17./18. Jahrhundert mögen zu ihrer Zeit als äquivalente Texte gegolten haben - der Zeitgeschmack forderte nun einmal die rigorose Adaptation der Dramenwelt und ihrer Figuren an die französische zeitgenössische Umwelt (vgl. G. Mounin, 1967, 37 ff.) -; heute sehen wir in diesen Übersetzungen eher Travestien als Texte, die Äquivalenz zu ihren Originalen aufweisen. Über Äquivalenz zwischen AS- und ZS-Text kann man demnach immer nur unter Bezugnahme auf die Entstehungszeit und die Entstehungsbedingungen einer Übersetzung diskutieren.

Aber nicht nur im Blick auf die Vergangenheit gilt die Flexibilität des Äquivalenzbegriffs. P. Newmark (1981, 156 f.) führt aus, daß der Standardbegriff aus dem Deutschen "der Klub der fünf Weisen" noch nicht in dieser Form ins Englische übersetzt werden kann und fährt fort: "A version such as "West Germany's Committee of top economic experts", known as "the Club of the Five Wise Men" may lead to a later literal translation." Zum gegebenen Zeitpunkt muß diese Übersetzung - eine "erklärende" Übersetzung - als äquivalent gelten, denn nur durch die Explikation gewinnt sie für den englischen Leser den kommunikativen Wert, die Verständlichkeit, welche die deutsche Entsprechung für den deutschen Leser hat. Sobald einmal die Engländer mit dieser Institution in der BRD vertraut sind, wird die Explikation überflüssig; "the Club of the Five Wise Men" wird als äquivalente Sprachzeichenmenge gelten können. So möchten wir auch nicht, wie W. Wilß (1980, 177), von "NullÄquivalenz" sprechen, wenn die englische lexikalische Einheit "floating voter" im Deutschen mit "Wechselwähler" wiedergegeben wird. Wilß bezeichnet solche Erscheinungen als "als Eins-zu-Null-Entsprechung(en) auf der langue-Ebene zu bewertende Lücke(n)", die nur durch adaptives Übersetzen kompensiert werden können. Wir möchten dem entgegenhalten, daß hier der Äquivalenzbegriff der kontrastiven Linguistik (langueorientiert) mit dem der Übersetzungswissenschaft (parole-orientiert) verwechselt wird. Durch "adaptives" Übersetzen, eine unter vielen ande- 
ren Übersetzungsprozeduren, wird hier eben Textäquivalenz erreicht, wenn das Lexem in einem Text verwendet wird.

Bei der Erörterung der Beispiele wurde der entscheidend wichtige Begriff der Funktion bereits eingeführt, ohne den Textäquivalenz nicht zureichend beschrieben werden kann. Aus der modernen Sprachwissenschaft wissen wir, daß dieselben sprachlichen Äußerungen je nach der Äußerungssituation unterschiedliche kommunikative Funktionen haben können ("Das ist ein bissiger Hund!" als Warnung oder als objektive Aussage oder gar als Anpreisung). Dieselben sprachlichen Elemente in einem Text können ebenso unterschiedliche Funktionen im Textganzen erfüllen. Die Alliteration in dem Textsegment

(7) Nacht ist wie ein stilles Meer / Lust und Leid und Liebesklagen / kommen so verworren her / in dem linden Wellenschlagen ... (Eichendorff)

hat die Funktion, den Wellenschlag zu symbolisieren, sinnlich erfahrbar zu machen. In dem Werbeslogan dagegen

(8) Milch macht Müde munter

hat die Alliteration die Funktion, Aufmerksamkeit zu erregen, einprägend zu wirken, positive Assoziationen auf das Produkt, für das geworben wird, zu übertragen. Bei einer äquivalenten Übersetzung müßte bei (7) der Symbolwert erhalten bleiben, bei (8) die leichte Einprägsamkeit, positive Assoziationen. Die sprachlichen Mittel, um das zu erreichen, werden den Möglichkeiten der Zielsprache entnommen, z.B. für (8) im Spanischen:

(8a) Leche - deleite de listos

- líquido de listos

Ein zweites Beispiel zur Verdeutlichung:

(9) Si yo digo "el sol sale por Oriente" lo que mis palabras ... propiamente dicen es que un ente de sexo varonil y capaz de actos espontáneos ... ejecuta la acción de salir. (Ortega; Hervorh. K.R.)

In einer äquivalenten Übersetzung wird "el sol" natülich im Deutschen mit "die Sonne" wiedergegeben und "un ente de sexo varonil" mit "ein Wesen weiblichen (und nicht: männlichen) Geschlechts", denn in dem Ortega-Essay fungiert dieses Textelement lediglich als Beispiel dafür, daß sich in der indogermanischen Satzstruktur eine Deutung der Wirklichkeit spiegelt, für die alles, was in der Welt geschieht, die Handlung eines geschlechtsbegabten Handelnden ist. Käme dasselbe Element - el sol, un ente de sexo varonil - in einem Text vor, der sich mit Götterund Heldensagen aus der Antike befaßt, in denen die Sonne als männli- 
ches Wesen - als Sonnengott und nicht -göttin - eine so große Rolle spielte, so müßte die Funktion des Segments als Ausdruck mythologischer Vorstellungen für eine äquivalente Übersetzung maßgebend sein. "Der Sonnengott" für "el sol" wäre im Deutschen textäquivalent, es wäre im Zusammenhang mit Göttersagen für den deutschen Leser nicht befremdlich, während dieselbe Übersetzungslösung im Ortega-Text: "der Sonnengott geht im Osten auf" lächerlich klänge.

\section{3. Äquivalenzkriterien}

Zwei Prinzipien bestimmen demnach die Aufstellung von Äquivalenzkriterien für die Übersetzung eines Textes. 1. Das Prinzip der Selektion und 2. das Prinzip der Hierarchisierung. Die Selektion wird vom Übersetzer vorgenommen, wenn er bei der AS-Textanalyse die für den jeweiligen Text merkmalhaften Elemente eruiert; die Hierarchisierung betrifft die Vorrangigkeit beizubehaltender Elemente, wenn nicht alle zugleich in der Zielsprache äquivalent gehalten werden können. Dabei sind jeweils die Funktion der einzelnen Textelemente in ihrem Beitrag zum Sinn des Gesamttextes und die Funktion des Textes selbst im Kommunikationsgeschehen die ausschlaggebenden Gesichtspunkte. Vgl. Bsp. (7) vs. Bsp. (8)

Alliteration - selektiertes merkmalhaftes Element

Sowohl die Funktion der beiden Texte als auch die Funktion der Elemente in den Texten sind verschieden: (7) expressiver (künstlerisch organisierter) Text, (8) operativer (persuasiv gestalteter) Text; Alliteration in (7) sprachkünstlerisches Element mit Symbolfunktion; Alliteration in (8) appellatives Element mit Persuasionsfunktion.

Die beiden erwähnten Funktionssorten sind in jedem individuellen Text zu ermitteln, wobei die objektiv gegebenen, materialiter vorhandenen Sprachzeichen als "Steuerungssignale" zur Feststellung der Funktionen einzelner Elemente dienen und die Textsorte und der Texttyp als Erkennungs- und Steuerungssignale für die Funktion des jeweiligen Textes im Kommunikationsgeschehen bestimmend sind. Die in der Zielsprache zu wählenden (bzw. gewählten) Sprachzeichen sind als äquivalent zu beurteilen, wenn sie analoge Steuerungs- und Erkennungssignale für den Zielleser aufweisen. Der Übersetzer analysiert den AS-Text, um den Einfluß der unterschiedlichen Faktoren, die den Übersetzungsprozeß beeinflussen (vgl. das Faktorenmodell in Reiß/Vermeer 1984, 148) - Sender, Empfänger, Sprache im Text, Text, -sorte, -typ, Situationskontext, soziokulturelle Einbettung - auf die Wahl der materialiter dargebotenen, und 
gerade dieser, Sprachzeichen zu untersuchen und den Textsinn zu erschließen (hermeneutischer Prozeß). Der AS-Text hat, semiotisch gesehen, wie jedes Zeichen drei Dimensionen: eine syntaktische, eine semantische und eine pragmatische.

Diese drei Dimensionen manifestieren sich auf verschiedenen sprachlichen Ebenen, angefangen vom Graphem/Phonem über Morpheme, Lexeme, Semanteme bis zur Textebene. Alle tragen zum Textsinn bei. Der Text hat Inhalt, Form und Funktion (im Kommunikationsgeschehen), die gemeinsam seinen Sinn ausmachen. Dabei werden Inhalt und Form von der Funktion dominiert. (Im Konversationslexikon werden unter dem Stichwort "Rabe" andere Inhaltselemente und eine andere sprachliche Form als angemessen vom Autor ausgewählt als in dem Gedicht "The Raven" von E.A. Poe. Die Funktion des ersten Textes ist es, Informationen sachlicher Art über den Redegegenstand "Rabe" zu vermitteln; die Funktion des zweiten Textes ist es, eine dichterische Vision des Redegegenstandes "Rabe" zu vermitteln und dies durch künstlerische Organisation der dazu ausgewählten Sprachzeichen).

Die Form betrifft einerseits die Textgestalt (= Textform) und dabei eingehaltene oder durchbrochene Konventionen/Normen im Ganzen; die Form betrifft andrerseits den Stil (= Sprachform) als Individualstil und als Funktionalstil. Textsinn manifestiert sich aber nicht nur in Inhalt und Form der einzelnen Sprachzeichen und des Gesamttextes. Auch paralinguale Elemente wie Rhythmus, Intonation und Versmaß (prosodische Elemente) können Funktion und Sinn mitbestimmen.

(10) Hast du auch gelesen, was da geschrieben steht? ("auch" = illokutiver Indikator)

(10a) Hast du auch gelesen, was da geschrieben steht? ("auch" als Semantem)

Sogar mit der Interpunktion (vgl. P. Newmark 1981, 171 f.) und mit Klein- und Großschreibung kann Textsinn "transportiert" werden.

Die in allen Texten vorkommenden Elemente - Syntagmen, Lexeme, Morpheme, Grapheme/Phoneme - haben zudem denotative und konnotative Bedeutungen und darüber hinaus die Fähigkeit, Assoziationen auszulösen, die alle zur Textfunktion beitragen und einen erheblichen Unsicherheitsfaktor bei der Textanalyse darstellen können, weil Konnotationen nicht übereinzelsprachlich und Assoziationen oft völlig individuell sind.

Es dürfte einleuchtend sein, daß beim kommunikativen Übersetzen angesichts der divergierenden Strukturen zweier Sprachen, der divergierenden Situationskontexte zweier Texte, der divergierenden soziokultu- 
rellen Gegebenheiten in zwei Sprachgemeinschaften die Sprachzeichen des AS-Textes nur in Ausnahmefällen einfach substituiert werden können. In der Regel ist eine Neuordnung der Relationen zwischen Inhaltsund Formelementen unter funktionalen Gesichtspunkten notwendig, damit der Zieltext in der Zielgemeinschaft dieselbe kommunikative Funktion erfüllen kann. Wenn Textäquivalenz erzielt werden soll, so wäre idealiter funktionale Äquivalenz zwischen allen einzelnen Textelementen in AS- und ZS-Text anzustreben; eine Forderung, die nicht einmal in der Translationstheorie für diesen Übersetzungstyp allen Ernstes erhoben wird. Daher ergibt sich die Notwendigkeit, die Äquivalenzkriterien mit Rücksicht auf Textsorte und Texttyp zu hierarchisieren. Bei der AS-Textanalyse wird untersucht, für welche Textelemente vorrangig Äquivalenz im Zieltext zu suchen ist. Die Hierarchie der einzelnen Werte kann, wie im Vorstehenden angedeutet, von Text zu Text (je nach Typ, Sorte und Individuum) wechseln. Bei einem philosophischen Traktat wird in erster Linie die Beibehaltung des Gedankengangs für die Äquivalenz des ZSTextes relevant sein; bei einem Kochrezept die korrekten Angaben für die Ingredienzien und die unmißverständliche Information über die Verarbeitung der Zutaten; bei einem Geschäftsbrief der Inhalt und die Textsortenkonventionen (z.B. bezüglich Anrede- und Schlußformeln); bei einem Gedicht die künstlerische Organisation des Textes; bei einem Werbespot die persuasiven Sprachelemente etc. Alle anderen Äquivalenzbestrebungen richten sich jeweils danach, in welcher Weise funktionale Äquivalenz einzelner Elemente zur Textäquivalenz beitragen können und müssen.

Dazu wieder einige Beispiele.

Bei den bisherigen Beispielen wurde bereits - unter anderem Gesichtswinkel - darauf verwiesen, daß u.U. ein bestimmtes Textelement mit Vorrang vor anderen in seiner Funktion äquivalent gehalten werden muß, um Textäquivalenz zu erzielen.

In dem bereits erwähnten Gedicht von E.A. Poe "The Raven" lautet der Refrain "nevermore". Er wurde von Poe wegen seines dunklen, unheilschwangeren Phonems /o/ und wegen des gleitenden Phonems /r/ gewählt (vgl. Radó 1979). Nach Aussage des Autors sind also diese Phoneme ausschlaggebend gewesen für die Wahl des Lexems "nevermore". Bei der Übersetzung müßte also neben der Semantik des Lexems auch die Phonetik in der Zielsprache erhalten bleiben, um kommunikativ äquivalent zu sein. Das ist nun nicht in allen Sprachen möglich. In der deutschen Übersetzung steht das Lexem "nimmermehr"; es ist semantisch, 
aber nicht phonetisch äquivalent - mit dem fehlenden dunklen /o/ gehen auch seine Assoziationen verloren -. Doch kann dieselbe Intonation: névermòre $=$ nímmermèhr, sowie die Lautähnlichkeit der Silben -more/mehr den Verlust einigermaßen kompensieren. Die mit diesem Refrain ausgelöste Assoziation des Unheilschwangeren wird vom zielsprachigen Leser eher durch die Assoziation des Tödlich-Endgültigen bei der steten Wiederholung des "nimmermehr" (mit seinem hämmernden Klang) ersetzt - keine invariante, aber eine analoge, der Funktion des Gesamttextes angemessene Assoziation. Bei der Übersetzung ins Französische - dort heißt es "jamais plus" - ist die semantische Komponente äquivalent, die phonetische und prosodische kann es, aufgrund der Verschiedenheit der französischen Sprache auch nicht entfernt sein. In diesem Fall geht die semantische Komponente bei der Erzielung der Sinnäquivalenz vor. Der Übersetzer wählt den wörtlichen Übersetzungstyp oder kann - in einer kommunikativen Übersetzung - die fehlende phonetische Äquivalenz durch ein "versetztes Äquivalent" (Kloepfer 1976, 167) an einer anderen Stelle, wo es sich von der Zielsprache her anbietet, kompensieren.

Eine Hilfe für die Hierarchisierung der Äquivalenzforderungen wird zuweilen im Text selbst gegeben.

(11) Das ästhetische Wiesel

Ein Wiesel / saß auf einem Kiesel / inmitten Bachgeriesel. (Chr. Morgenstern)

(11a) A weasel / perched on an easel / within a patch of teasel. (Ü.: M. Knight)

Hier sind im Zieltext syntaktische und semantisch-pragmatische Äquivalenz gegeben und damit Textäquivalenz erreicht, obwohl lexikalischsemantische Änderungen vorgenommen wurden, um der künstlerischen Intention (die zur Funktion des Textes wird), gerecht zu werden. Das künstlerische Organisationsprinzip wird vom Autor selbst im letzten Vers genannt und damit für den Übersetzer zur vorrangig zu beachtenden Äquivalenzforderung:

(11b) Das raffinier- / te Tier / tat's um des Reimes willen.

Jin Levy (1969), dem dieses Beispiel entnommen ist, erwähnt noch weitere fünf Übersetzungsmöglichkeiten, in denen von M. Knight jeweils andere lexikalisch-semantische Veränderungen vorgenommen werden, die höchstrangige Äquivalenzforderung aber jeweils erfüllt wurde, weshalb Levy alle Lösungen als äquivalent beurteilt (ein weiterer Hinweis auf die Flexibilität und Bandbreite des Begriffs der Textäquivalenz).

(12) Sur les faire-part on avait précisé "ni fleurs ni couronnes", histoire de ne pas 
mettre les copains dans les frais..." (San Antonio, De "A" jusqu'à "Z", Paris 1967)

(12a) Auf den Traueranzeigen hatte gestanden "weder Blumen noch Kränze", um die lieben Freunde nicht in Unkosten zu stürzen ... (Ü.: S.M. Roediger 1973)

In dieser Textpassage wird eine in französischen Todesanzeigen übliche Formulierung (Textsortenkonvention) zitiert: "ni fleurs ni couronnes". In deutschen Todesanzeigen heißt diese Formulierung üblicherweise etwa: "Von Blumen- und Kranzspenden bitten wir abzusehen". Die Übersetzung mit "weder Blumen noch Kränze" ist pragmatisch nicht äquivalent, sie ist für den deutschen Leser zumindest befremdlich; im Unterschied zu seinen Erwartungen, wenn von einer Todesanzeige die Rede ist, wirkt das Textsegment, wenn er es überhaupt richtig einordnen kann, auf ihn eher unhöflich, was die kommunikative Äquivalenz zumindest stark beeinträchtigt. Ein einzelner Fehlgriff in einem ganzen Roman wird nun kaum die gesamte Textäquivalenz zunichte machen, häufen sich jedoch in einer Übersetzung solche Fälle, so ist die Textäquivalenz insgesamt gefährdet.

Einleuchtend dürfte es sein, daß die folgende, von v. Stackelberg $(1978,95)$ positiv bewertete Übersetzung nicht als äquivalent bezeichnet werden kann, weil sie trotz aller Stiläquivalenz an entscheidender Stelle mit einem geänderten Inhaltselement auch alle an dieses geknüpfte Assoziationen verändert.

(13) La nuit ... Sied-il bien à des Dieux de dire qu'ils sont las?

Mercure ... Les Dieux sont-ils de fer? (Molière, Amphitryon)

(13a) Die Nacht ... Sich müd zu nennen, ziemt das einem Gott?

Merkur ... Sind Götter denn von Stein? (Ü.: A. Luther)

Es geht nicht darum, ob Götter gefühllos (= Stein) sind, sondern darum, ob sie zugeben dürfen, auch einmal von übermäßiger Anstrengung müde geworden zu sein.

(13b) Sind Götter denn von Stahl! (Ü.: Fulda)

Das Inhaltselement "fer" ist auch hier abgewandelt; Eisen und Stahl sind nicht dasselbe. Aber in der Sprachverwendung (französisch und deutsch) sind sie im Blick auf die auszulösenden Assoziationen und im Rhythmus (was für den vorliegenden Texttyp wichtig ist) gleichwertig, d.h. äquivalent.

(14) Ein jeder Engel ist schrecklich (R.M. Rilke, 1. Duineser Elegie)

(14a) Each single angel is terrible. (Ü.: Leishman/Spender 1952)

Zwischen diesen beiden Textsegmenten besteht auf den ersten Blick Äquivalenz, die bis auf die Wortebene hinabreicht. Für einen informa- 
tionsvermittelnden Text (etwa die Übersetzung einer Beschreibung von mißglückten Engelsskulpturen an einem Gebäude, z.B. in einem Reiseführer) ließe sich dieses Urteil durchaus aufrechterhalten. Nicht so im Hinblick auf den gesamten sprachlichen Kontext, in dem dieses Textsegment steht. Die Elegie beginnt mit den Zeilen: "Wer, wenn ich schriee, hörte mich denn aus der Engel / Ordnungen? Und gesetzt selbst, es nähme / einer mich plötzlich ans Herz: ich verginge von seinem / stärkeren Dasein, denn das Schöne ist nichts / als des Schrecklichen Anfang ..." Hier wird die Dimension des Numinosen evoziert, der im Englischen die triviale Formulierung "is terrible" im angeführten Textsegment nicht gerecht werden kann.

(14b) Round every angel is terror. (Ü.: Wydenbruck 1948)

dagegen erreicht Äquivalenz auf der Textebene durch Berücksichtigung des gesamten Kontextes.

(15) One doesn't miss what one has never had; but it's awfully hard going without things after one has commenced thinking they are his-hers (English language needs another pronoun) by natural right. (Webster, Daddy-Long-Legs 1967, 126)

(15a) Was man nie gehabt hat, vermißt man nicht, aber es ist entsetzlich, ohne Dinge zu leben, die man für ein natürliches Anrecht hält. (Ü.: M. Boveri, 1979, 145)

(15b) Was man nie besaß, das wird man auch nicht vermissen; aber es ist ungemein schwer, im späteren Leben ohne den gewohnten Genuß zu leben, nachdem man sich schon daran gewöhnt hatte, zu glauben, man besäße diese Dinge von Rechts wegen. (Ü.: M. Boesch-Frutiger, 1970, 177 f.)

Im Blick auf die (von uns hervorgehobenen) Elemente des vorliegenden Textsegments könnte man Äquivalenz zwischen dieser Passage in AS- und ZS-Texten verneinen. "His-hers" ist mit derselben sprachlichen Einheit "man" wiedergegeben wie das Element "one", und die metasprachliche Äußerung in der Klammer fehlt in beiden ZS-Texten ganz. Trotzdem sind beide Texte (in diesem Punkt!) als dem AS-Text äquivalent anzusehen, denn einmal lassen die unterschiedlichen Strukturen der Ausgangs- und der Zielsprache eine solche metasprachliche Äußerung in der Übersetzung als paradox erscheinen (das Deutsche hat das Pronomen ja zur Verfügung, dessen Fehlen im Englischen hier denunziert wird: "they are his-hers" = "daß sie einem gehören"), zum andern hat sie für den Zielleser in der vorliegenden Textsorte (Jugendroman) keinen kommunikativen Wert. Anders würde es sich verhalten, wenn diese Elemente z.B. in einer Passage eines kontrastiv orientierten englischen Lehrwerks enthalten wären, dessen Funktion u.a. darin besteht, über die unterschiedlichen Strukturen der englischen Sprache und eventuelle "Lücken im 
System" zu informieren. Die aus dieser Erörterung zu ziehende Folgerung ist demnach, daß Äquivalenz zwischen ausgangssprachlichen und zielsprachlichen Textelementen nicht ein für allemal, auch nicht "sprachenpaarbezogen", festgestellt werden kann und daß Textäquivalenz nicht nur in Relation zum Phänomen Text zu sehen ist, sondern auch vom Phänomen Textsorte bzw. Texttyp abhängt.

(16) Je m'adresse aux peuples, aux peuples au pluriel. (aus einer Ansprache De Gaulles)

(16a) Ich wende mich an das Volk, an die Völker.

(16b) Ich wende mich an die Völker.

Beide Übersetzungen weisen u.U. Äquivalenz zum Ausgangstext auf; jedoch (16a) nur für das Dolmetschen, (16b) für das Übersetzen. Der Dolmetscher, der die Passage hörend aufnimmt, kann beim ersten "aux peuples", aufgrund der Gesetze der französischen Phonetik, noch nicht wissen, daß der Plural "gemeint" ist, weshalb der Redner ja auch das Textelement noch einmal aufnimmt und durch "au pluriel" vereindeutigt; was der Dolmetscher, wenn er "zu schnell" reagiert, ebenso tun wird. Wird diese Rede z.B. auf Tonband fixiert und später schriftlich übersetzt, so würde (16a) eine falsche Information ("an das Volk" = "an das französische Volk" ist nicht Bestandteil des AS-Textes) transferieren; eine wörtliche Übersetzung: "an die Völker, an die Völker im Plural" dagegen ergäbe in der Zielsprache eine im Ausgangstext nicht vorhandene Redundanz, wobei der Zusatz "im Plural" entweder als unverständlich oder im günstigsten Fall als (im Original nicht enthaltene) Emphase aufgefaßt würde, dem Text also Elemente hinzufügte, denen kein äquivalentes Element im Ausgangstext gegenübersteht.

\section{Zusammenfassung}

Aus der bisherigen Darlegung geht hervor, daß es Äquivalenz zwischen AS-Text und ZT-Text auf verschiedenen Ebenen eines Textes als Kommunikationsmittel gibt. Die einzelnen Elemente auf den verschiedenen Ebenen können aufgrund der Verschiedenheiten der Sprachen und der sozio-kulturellen Einbettung der Kommunikation in den meisten Fällen nicht invariant und nicht alle zugleich äquivalent gehalten werden. Dann stellt der Übersetzer fest, welche Elemente des Ausgangstextes er für den konkret vorliegenden Text als merkmalhaft, d.h. funktional relevant, auswählt (Prinzip der Selektion), und in welcher Reihenfolge er die Beachtung der Merkmale für vordringlich hält (Prinzip der Hierarchisierung). Danach richtet sich die Entscheidung, in welchen Fällen er auf eine äquivalente Wiedergabe des jeweiligen Merkmals verzichten will (oder muß), in welchen Fällen er Kompensationen (versetzte Äquivalen- 
te) und in welchen Fällen er die Reproduktion des entsprechenden Elements wählen will (oder muß), um für den Zieltext insgesamt Äquivalenz, d.h. Gleichwertigkeit in bezug auf die Funktion des Textes im Kommunikationsgeschehen innerhalb der Zielgemeinschaft zu erreichen. Bei diesen Entscheidungen, die immer die Funktion einzelner Elemente im Gesamttext berücksichtigen - die Fähigkeit dazu ist das, was man translatorische Kompetenz im Blick auf kommunikatives Übersetzen nennt - helfen ihm der jeweilige sprachliche Kontext, der Situationskontext, die sozio-kulturelle Einbettung des jeweiligen Textes, seine Zugehörigkeit zu einer Textsorte und zu einem Texttyp, die beide Rückschlüsse auf die Funktion des Textindividuums im Kommunikationsgeschehen erlauben. Abhängig sind die jeweiligen Entscheidungen auch von den Strukturverschiedenheiten des jeweils involvierten Sprachenpaars.

Damit erweist sich Äquivalenz als ein flexibler Begriff für eine Relation zwischen einem AS- und einem ZS-Text, die bei jedem Text (und jedem seiner Elemente in seinem Beitrag zum Sinn dieses Textes) eine Neuordnung der Relevanz aller jener Faktoren erfordert, die den Übersetzungsprozeß beeinflussen. Selektion und Hierarchisierung dieser Faktoren erfolgen durch den Übersetzer und machen einen Teil seiner translatorischen Kompetenz aus, die weit über die bloße Beherrschung der involvierten Sprachen hinausgeht. Die unumgängliche Subjektivität dieser Selektion und Hierarchisierung ist nicht mit Beliebigkeit gleichzusetzen; sie wird gesteuert von den im Text manifesten Sprachzeichen und den objektiv den Übersetzungsprozeß (in Analyse- und Reverbalisierungsphase) determinierenden Faktoren. Eben dies ermöglicht es, ein Phänomen wie Äquivalenz zu beobachten, auf argumentativem Wege intersubjektiv nachvollziehbar $\mathrm{zu}$ beschreiben und $\mathrm{zu}$ beurteilen, den Begriff der Äquivalenz mit einem definierten Inhalt zu füllen. Äquivalenz zwischen AS- und ZS-Text besteht in der je gleichwertigen Relationierung von Inhalt(en) und Form(en) eines Textes in ihren Funktionen zur Erreichung des Textsinns.

\section{Literatur}

Kloepfer, Rolf: Die Theorie der literarischen Übersetzung. München 1967

Levy, Jiri: Die literarische Übersetzung. Theorie einer Kunstgattung. Frankfurt/M.-Bonn 1969

Mounin, Georges: Die Übersetzung. Geschichte, Theorie, Anwendung. München 1967

Newmark, Peter: Approaches to Translation. Oxford 1981

Radó, G.: Outline of a systematic translatology, in: BABEL 25, 1979, 187-196

Reiss, Katharina / Vermeer, Hans J.: Grundlegung einer allgemeinen Translationstheorie. Tübingen 1984

Stackelberg, Jürgen von: Weltliteratur in deutscher Übersetzung. Vergleichende Analysen. München 1978

Toury, Gideon: In search of a theory of translation. Tel Aviv 1980

Wilss, Wolfram: Übersetzungswissenschaft. Probleme und Methoden. Stuttgart 1977 
